\title{
Mechanical strength of photonic crystal fibres
}

\author{
Michał A. Matusewicz, Jan Wójcik, ${ }^{*}$ Paweł Mergo, and Aleksander Walewski \\ Faculty of Chemistry, Maria Skłodowska-Curie University, M. Skłodowska-Curie Sq. 3, 20-031 Lublin
}

Received March 18, 2010; accepted March 25, 2010; published March 31, 2010

\begin{abstract}
Most of the glass fractures start from flaws on the glass surface. The outer surface of optical fibres is protected from the influence of mechanical and chemical factors by a thin layer of polymer protective coating. Photonic crystal fibres have, besides outer glass surface, inner glass surface of even an order of magnitude greater area. This inner surface is not exposed to mechanical factors. However, it is not protected from chemical agents, most importantly - water vapour. The hypothesis of relatively lower mechanical reliability of photonic crystal fibres in comparison to classical optical fibres was initially confirmed in our previous works.

We present the work containing a quantitative mechanical strength description of two kinds of optical fibre mentioned above.
\end{abstract}

Photonic crystal fibres (PCF) are introduced to a constantly growing range of applications, most notably in various sensors. This requires deep knowledge about PCF properties, including their mechanical reliability. This problem is well known and described [1,2] for classical fibres. To the best of our knowledge, no work has been describing mechanical reliability of photonic optical fibres.

Compared to conventional fibres, PCF have a significant inner glass surface, a smaller glass surface area in the fibre's cross-section and a much more complicated manufacturing process. All those facts result in a higher probability of a defect occurrence. Thus, in our previous publication [3] we suggested that photonic crystal fibres should have lower mechanical reliability than classical fibres of the same diameter.

Here we discuss the strength of two fibres: conventional optical fibre and photonic crystal fibre.

A well known fact is that most of the flaws creating cracks - starting points of fibre - breaking is located on the glass surface. The classic Griffith's criterion[4] states that the square root of the crack's length is inversely proportional to the fracture stress. Crack growth is caused by mechanical and chemical factors. Most notable of chemical factors weakening optical fibres is water vapour. It reacts with $\mathrm{SiO}_{2}$ groups giving silanol groups. If this process occurs inside the crack, chemical stress accelerating the crack growth is created [5]. The outer glass surface of an optical fibre is protected from

$$
{ }^{*} \text { E-mail: jan.wojcik@umcs.lublin.pl }
$$

chemical agents by polymer coating, but the internal glass surface in a PCF is exposed.

We test the mechanic strength of optical fibres on a Tira Test 2200 tensile testing machine. A $500 \mathrm{~mm}$ long fibre sample was stretched at the rate of $50 \mathrm{~mm}$ per minute. Stress is calculated for a PCF for the effective glass surface area. The results are presented as histograms showing the distribution of fracture stress value (Fig. 1, Fig. 2). It may be observed that in the case of photonic crystal fibre fractures occur in a wider range of stress values than for a conventional fibre, including isolated cases of very low stress.

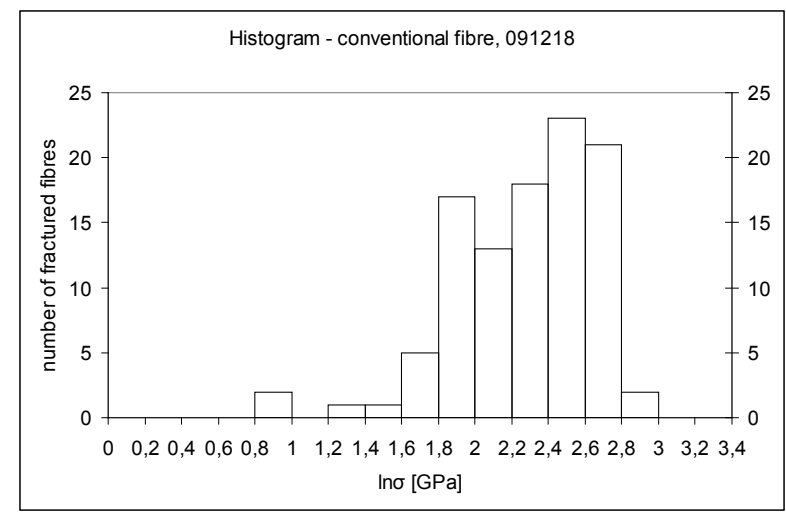

Fig. 1: Histogram showing the number of classical fibre samples breaking in defined stress ranges.

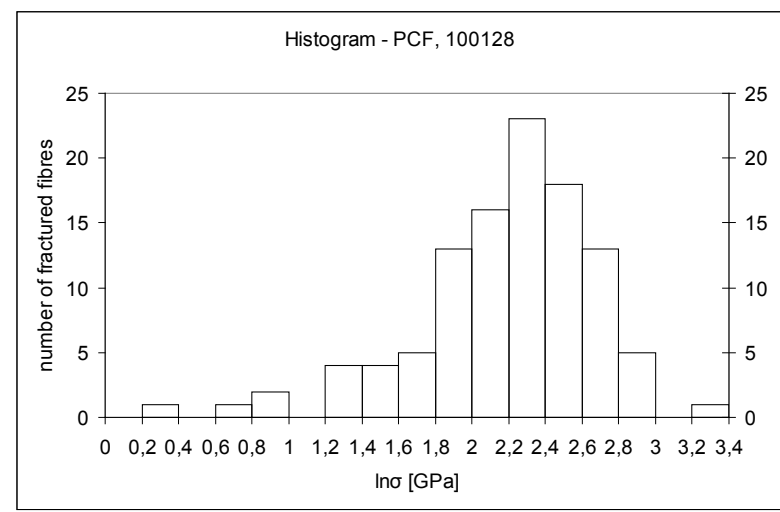

Fig. 2: Histogram showing the number of PCF samples breaking in defined stress ranges. 
Obtained results are presented also as the Weibull distribution plots given by the following equation:

$$
\ln \ln \left(\frac{1}{1-F_{k}}\right)=\ln L+m \ln \sigma
$$

where $F_{k}$ is the probability of breaking a fibre of length $L$ subjected to stress $\sigma$. The Weibull coefficient, being the distribution slope, is denoted as $m$. As shown in the Fig. 3 and Fig. 4, the values of the Weibull coefficients are relatively high, which signifies the wear-out character of failures. For the photonic crystal fibre $m$ is 6.16 and for the classical fibre it is 6.64 . The difference may be caused by a very complex PCF manufacture process in which glass is subjected to numerous temperature changes.

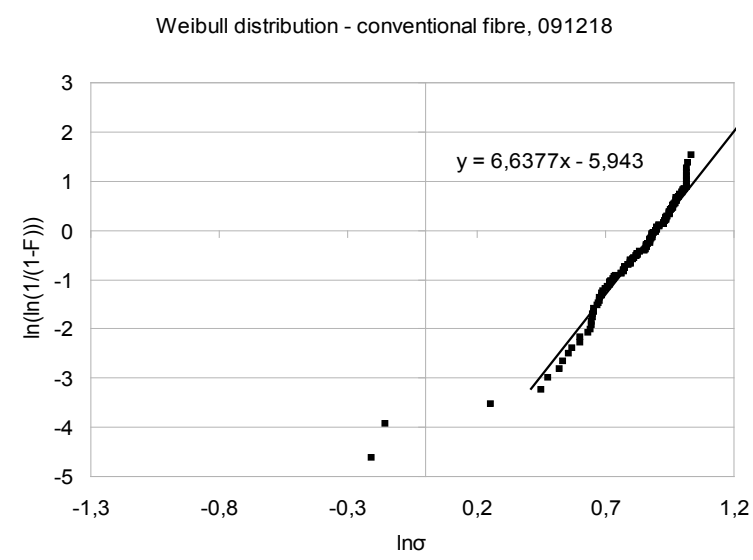

Fig. 3: Weibull distribution of results obtained for classical fibre.

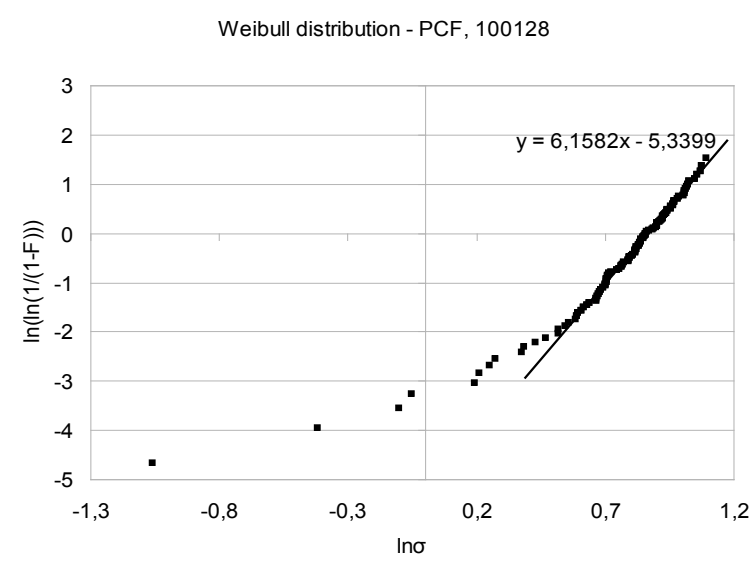

Fig. 4: Weibull distribution of results obtained for $P O F^{*}$

As an additional tool for finding the exact location of the flaw leading to the fracture fractography was used. Our intention was to check the assumption of multiple reasons of photonic crystal fibre breaking. However, an important drawback of broken fibres fractography is that only fibres fractured at the very low stress can be analysed.

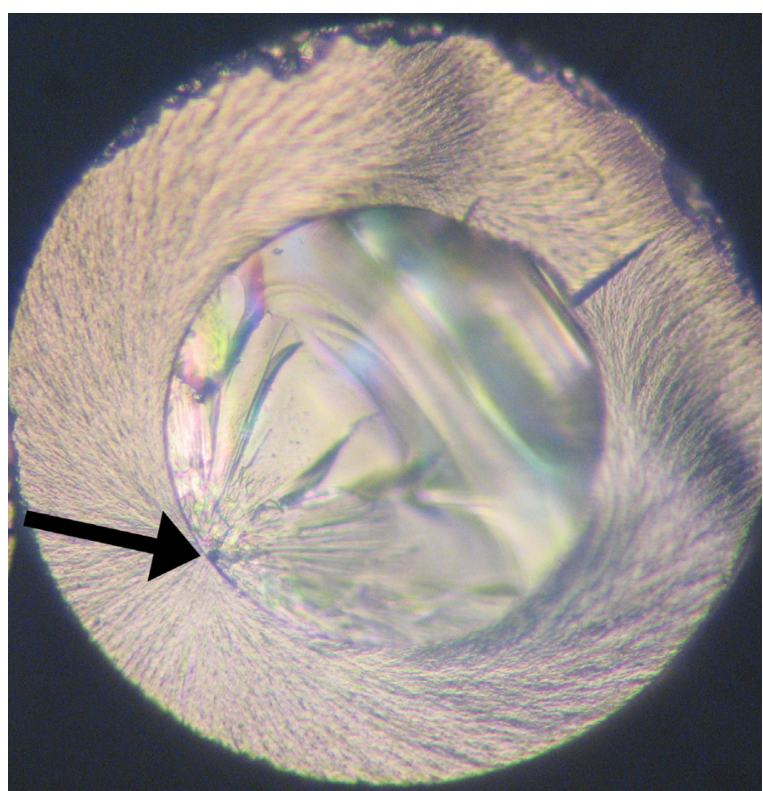

Fig. 6: Fractographic picture of broken conventional fibre. Flaw from which fracture started is indicated by an arrow.

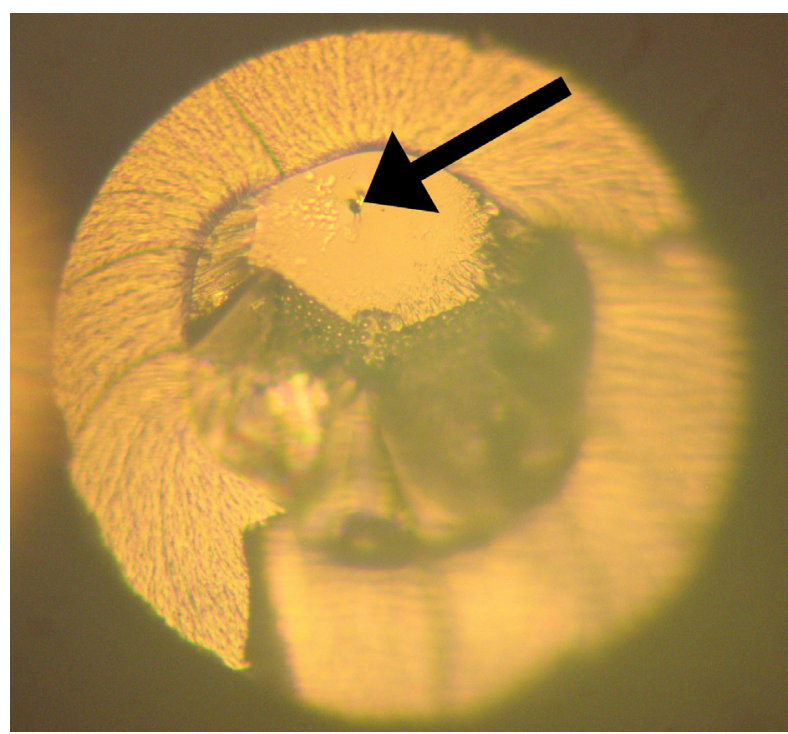

Fig. 5: Fractographic picture of a broken PCF. The flaw starting the fracture, located in a part of the fibre stacked from rods, is pointed by an arrow.

Figure 5 shows an example of broken classical optical fibre. As expected, fractures start at the outer surface of the glass. In Fig. 6 a broken photonic crystal fibre is presented. The flaw being the starting point of a fracture is located inside the fibre. This PCF was manufactured with the stack and draw method, in which capillaries and 
rods are stacked together and subjected to further treatment. In the presented case, the flaw is placed in the zone of stacked rods. We suppose that this defect was created by impurity during an imperfect manufacture process. We also know that apart from the mechanism presented above, fractures of photonic crystal fibres may be caused by flaws in other zones of the microstructured fibre, as well as on the outer glass surface [3].

The present letter is, in fact, a preliminary attempt to compare the mechanical properties of conventional and photonic crystal optical fibres. The initial results confirm our hypothesis that photonic crystal fibres have lower mechanical strength than conventional fibres of the same diameter.
The present work is a part of the research carried out in the PHOSFOS project within the framework of the EU Seventh Framework Programme.

\section{References}

[1] Ch. R. Kurkijan, J. T. Krause, M. J. Matthewson, J. Lightwave Tech. 7, 1360 (1989).

[2] E. A. Lindholm, E. Warych, D. Whelan, Proc. SPIE 5663, 129 (2009).

[3] J. Wójcik, M. A. Matusewicz, P. Mergo, M. Makara, PAKGOŚ, 4, 7 (2009).

[4] A.A. Griffith, Philos. T. R. Soc. Lon., A 221, 163 (1921).

[5] T. A. Michalske, S. W. Freiman, J. Am. Ceram. Soc. 66(4), 284 (1983). 